\title{
COVID-19 in pediatric patients: a case series from the Bronx, NY
}

\author{
Einat Blumfield ${ }^{1}$ (D) $\cdot$ Terry L. Levin ${ }^{1}$
}

Received: 10 May 2020 /Revised: 22 June 2020 / Accepted: 9 July 2020 / Published online: 29 July 2020

(C) Springer-Verlag GmbH Germany, part of Springer Nature 2020

\begin{abstract}
Background Coronavirus disease 2019 (COVID-19) primarily affects adults, with a lower incidence in children.

Objective To report our experience with critically ill children with COVID-19.

Materials and methods We reviewed the medical records of children with COVID-19 who were admitted Feb. 25 to May 1, 2020. We reviewed patient demographics, symptoms, comorbidities, requirement for respiratory support, evidence of acute myocardial injury, and chest radiographs.

Results The study included 19 children and adolescents (ages 2 months to 18 years, median 8 years; 10 males, 9 females; 18 COVID-19-positive, 1 COVID-19-negative with positive exposure). Presenting symptoms included fever (89\%), cough (68\%), respiratory distress (68\%) and vomiting/diarrhea (47\%). Comorbidities were present in $12(63 \%)$. Fourteen required intensive care; eight required intubation. Two children died. Five patients developed acute myocarditis (median age 7 years); in all five, chest radiographs were notable for cardiomegaly and pulmonary congestion or interstitial edema. Of these five, one (age 18 years), who had underlying hypertension and obesity, developed multifocal pneumonia and renal failure. The other four were previously healthy; three (ages 5 years, 7 years, 8 years) were subsequently diagnosed with multisystemic inflammatory syndrome in children (MIS-C); one developed pulmonary opacities consistent with adult respiratory distress syndrome, three $(60 \%)$ had no parenchymal pulmonary opacities. Fourteen patients (median 13 years), most with comorbidities, had no acute myocardial injury. Chest radiographs in 13 (93\%) demonstrated parenchymal lung disease with a predominant perihilar and basilar distribution.

Conclusion Myocarditis without pulmonary disease occurred in children in their first decade as a component of MIS-C, a newly described syndrome of multisystemic inflammation requiring further investigation. Pulmonary disease dominated the radiographic features of COVID-19-positive adolescents in their second decade in whom radiographs demonstrated predominantly perihilar and basilar distribution of lung opacities.
\end{abstract}

Keywords Adolescents · Children · Coronavirus · COVID-19 $\cdot$ Heart $\cdot$ Multisystem inflammatory syndrome $\cdot$ Myocarditis · Pneumonia $\cdot$ Radiography

\section{Introduction}

Coronavirus disease 2019 (COVID-19), a disease caused by the severe acute respiratory syndrome coronavirus-2 (SARSCoV-2), was declared a pandemic by the World Health Organization on March 11, 2020 [1]. The infection has rapidly spread over the last months and led to more than 510,000 deaths around the world as of June 30 [2]. The highest

Einat Blumfield

eblumfie@montefiore.org

1 Department of Radiology, Division of Pediatric Radiology, Montefiore Medical Center,

111 E. $210^{\text {th }}$ St., Bronx, NY 10467, USA morbidity and mortality occur in older adults and in people with pre-existing medical conditions including hypertension, obesity and diabetes [3].

Most people infected with COVID-19 are either asymptomatic or present with a mild, self-limiting illness. However, 15-29\% of patients become critically ill with multifocal pneumonia and develop respiratory failure, necessitating mechanical ventilation $[1,3]$. Additional complications include acute renal failure in up to $15 \%$ of affected adults [4], thromboembolic disease secondary to platelet activation and endothelial damage [5], and myocardial injury [6].

Children infected by SARS-CoV-2 are often asymptomatic or develop only mild symptoms [7, 8]. However, in April a new syndrome of multisystemic inflammation in children (MIS-C) associated with COVID-19 emerged and was reported initially in the United Kingdom [9]. At our institution in the 
Bronx, NY, we have seen a large number of critically ill pediatric patients, including several young children with acute myocardial injury and MIS-C. In this manuscript, we report our unique experience with critically ill pediatric patients with COVID-19, including clinical and radiographic findings.

\section{Materials and methods}

Our institutional review board approved this retrospective study, for which we searched the picture archiving and communication system (PACS) for chest radiographs performed on pediatric patients (ages 0-18 years) with known or suspected COVID-19 infection admitted to our children's hospital between Feb. 25 and May 1, 2020. Children were excluded if they had a negative test for COVID-19 or were not clinically suspected of having COVID-19. Children presenting to the emergency room but not requiring hospital admission were excluded.

Using the electronic medical records of the identified patients, we collected the following data: patients' age, gender, presenting symptoms, underlying medical conditions, requirement for intubation and mechanical ventilation or other respiratory support, evidence of acute myocardial injury based on cardiology consults, and hospital course.

The chest radiographs of each child were reviewed by one of two certified pediatric radiologists with 15 years (E.B.) and 19 years (T.L.L.) of experience. They analyzed chest radiographs for the distribution and type of pulmonary opacities (interstitial, hazy, consolidation), cardiomegaly, pleural effusion, and pulmonary vascular congestion or pulmonary edema.

\section{Results}

Nineteen patients (age range 2 months to 18 years, median age 8 years) including 10 males and 9 females were admitted during the study period. Eighteen tested positive for COVID-19 using real-time reverse transcription polymerase chain reaction (RT-PCR) test; one tested negative (a 5-yearold boy who had close contact with family members with COVID-19 and presented with fever, headache, cough, vomiting and chest pain, developed myocarditis and was subsequently diagnosed with MIS-C).

Nine patients had a respiratory virus test panel performed. Seven were negative. Two (a 14-year-old and a 17-year-old) had positive test panels, one for rhinovirus and the second for a corona virus (non-SARS-CoV-2).

Presenting symptoms included fever in 17 (89\%), cough in $13(68 \%)$, respiratory distress in $13(68 \%)$, and vomiting or diarrhea in $9(47 \%)$. Less common symptoms included rhinorrhea/nasal congestion in $6(31 \%)$, headache in 3
(16\%), sore throat in $2(10 \%)$ increased seizure activity in 2 $(10 \%)$, and abdominal pain in $2(10 \%)$.

Comorbidities were present in 12 patients $(63 \%)$, including severe neurologic impairment in 5 , morbid obesity in 3 , as well as congenital heart disease, cardiomyopathy, metastatic cancer, asthma, hypertension, sickle cell disease, a history of prior thromboembolic events and Fragile X syndrome, each present in 1 patient. Some patients exhibited more than one comorbidity.

Fourteen patients required admission to the pediatric intensive care unit. Of these, eight required intubation and one with a known diagnosis of myasthenia gravis, tracheostomy and chronic respiratory failure continued pre-admission mechanical ventilation. Four required high-flow nasal cannula and one required bilevel positive airway pressure.

Five children $(26 \%)$ developed acute myocardial injury (median age 7 years). In all five, echocardiograms demonstrated either biventricular or left ventricular dysfunction with decreased ejection fraction. Troponin was elevated in three patients, ranging $0.16-0.27 \mathrm{ng} / \mathrm{mL}$ (normal range $<0.1 \mathrm{ng} /$ $\mathrm{mL}$ ) and N-terminal pro-brain natriuretic peptide (NTproBNP) was elevated in all patients, ranging from $500 \mathrm{pg} /$ $\mathrm{mL}$ to $>15,000 \mathrm{pg} / \mathrm{mL}$ (normal range $<125 \mathrm{pg} / \mathrm{mL}$ ). Of the five children with acute myocardial injury, three ( 5 years, 7 years, 8 years old) with no pre-existing conditions who presented with fever and gastrointestinal symptoms were subsequently diagnosed with MIS-C. In one of these children, an 8-year-old girl, echocardiogram demonstrated ectatic coronary arteries in addition to myocardial dysfunction. This child developed hypotensive shock and adult respiratory distress syndrome. A fourth patient (age 2 months) with no comorbidities presented with apnea, choking episode and respiratory distress with no fever and was diagnosed with myocarditis secondary to COVID-19. The fifth patient (age 18 years), with pre-existing hypertension and morbid obesity, developed parenchymal lung disease consistent with multifocal pneumonia that progressed to adult respiratory distress syndrome and renal failure in addition to myocarditis. Three of the five patients with acute myocardial injury (the 2-month-old, 8year-old with MIS-C and 18-year-old with COVID-19 pneumonia) required intubation and mechanical ventilation (Table 1).

Chest radiographs in the five patients with acute myocardial injury were notable for cardiomegaly and vascular congestion or interstitial pulmonary edema in all (Fig. 1). In three (60\%) of these children (a 2-month-old, 5-year-old and 7year-old) parenchymal pulmonary opacities were absent. Two of these children had small pleural effusions. The other two patients with myocarditis - an 18-year-old with COVID19 pneumonia and acute renal failure, and an 8-year-old with MIS-C - developed diffuse parenchymal lung opacities and a clinical picture consistent with adult respiratory distress syndrome (Fig. 2). 
Table 1 Comparison between patients with and without acute myocarditis

\begin{tabular}{llrr}
\hline & & $\begin{array}{l}\text { Acute myocarditis } \\
(n=5)\end{array}$ & $\begin{array}{l}\text { No acute myocarditis } \\
(n=14)\end{array}$ \\
\hline Demographics & Median age (years) & 7 & 13 \\
& Gender & $3 \mathrm{M}, 2 \mathrm{~F}$ & $7 \mathrm{M}, 7 \mathrm{~F}$ \\
$\begin{array}{c}\text { Pre-existing conditions and } \\
\text { clinical presentation }\end{array}$ & Pre-existing condition & $1(20 \%)$ & $11(79 \%)$ \\
& Fever & $4(80 \%)$ & $13(93 \%)$ \\
& Cough & $2(40 \%)$ & $11(78 \%)$ \\
& Respiratory distress & $4(80 \%)$ & $9(64 \%)$ \\
& GI symptoms & $3(60 \%)$ & $6(43 \%)$ \\
Severity of illness & High-flow nasal cannula & $2(40 \%)$ & $2(14 \%)$ \\
& Intubated & $3(60 \%)$ & $2(36 \%)$ \\
Madiographic findings & Mortalities & 0 & $2(14 \%)$ \\
& Cardiomegaly & $5(100 \%)$ & $2(14 \%)$ \\
& Congestive heart failure & $5(100 \%)$ & $1(7 \%)$ \\
& Pleural effusion & $3(60 \%)$ & $13(93 \%)$ \\
& Pneumonia/ARDS & $2(40 \%)$ & $1(7 \%)$ \\
\hline
\end{tabular}

$A R D S$ acute respiratory distress syndrome, $F$ female, $G I$ gastrointestinal, $M$ male
Fourteen patients (74\%) had no evidence of acute myocardial injury (median age 13 years). Of these, 11 (79\%) had a pre-existing condition, most frequently neurologic dysfunction (Table 1). Two patients from this group died during the study period; both had comorbidities.

Other than the two patients in this group who had preexisting cardiomegaly from sickle cell disease and congenital heart disease, respectively, none demonstrated cardiomegaly. One patient had pleural effusion and $13(93 \%)$ had parenchymal lung disease (Fig. 3). One child (age 3 months) had a normal chest radiograph despite respiratory symptoms.

Table 2 demonstrates the distribution and type of pulmonary opacities in the 15 patients with radiographic findings of parenchymal lung disease. Chest radiographs demonstrated pulmonary consolidation in $13(87 \%)$, hazy pulmonary

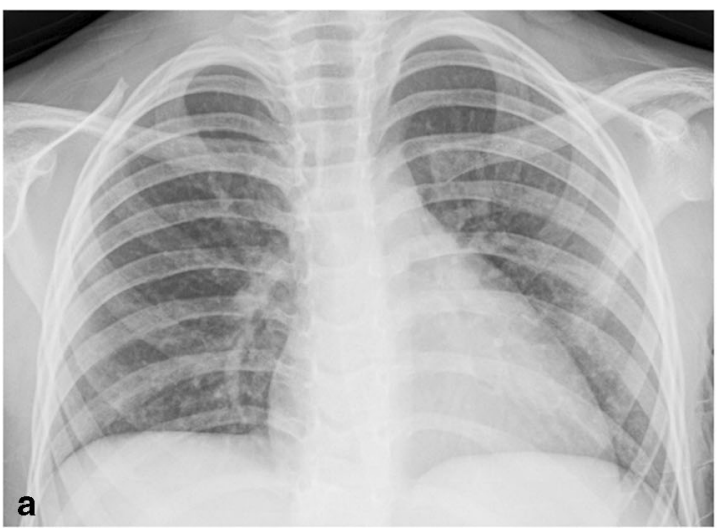

Fig. 1 Chest radiographs in a 5-year-old previously healthy boy with COVID-19 and myocarditis who was subsequently diagnosed with multisystemic inflammatory syndrome in children (MIS-C). a Admission anteroposterior (AP) chest radiograph. The cardiac silhouette is normal in size and the lungs are clear. b AP chest opacities in $8(53 \%)$ and interstitial opacities in $6(40 \%)$ patients. Ten (67\%) patients had more than one type of parenchymal opacity. The distribution of the opacities was as follows: perihilar in $11(73 \%)$, lower lobes in $9(60 \%)$, diffuse in $5(33 \%)$ and peripheral in $1(7 \%)$. Eight (53\%) patients had a combination of perihilar and lower lobe opacities.

Hilar and mediastinal adenopathy were absent in all patients in our cohort.

\section{Discussion}

While the pandemic of COVID-19 has severely affected the global community, the infection primarily affects adults, with a significantly lower incidence in children. As such, much of

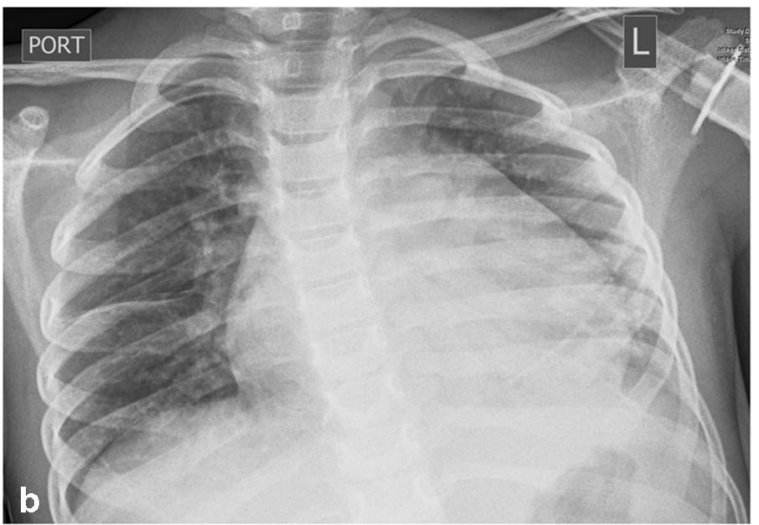

radiograph from hospital Day 3 demonstrates cardiomegaly and interstitial pulmonary edema with fluid in the minor fissure. There is left lower lobe atelectasis, likely secondary to the cardiomegaly. No other parenchymal opacities are present. Of note, respiratory viral panel testing was negative 


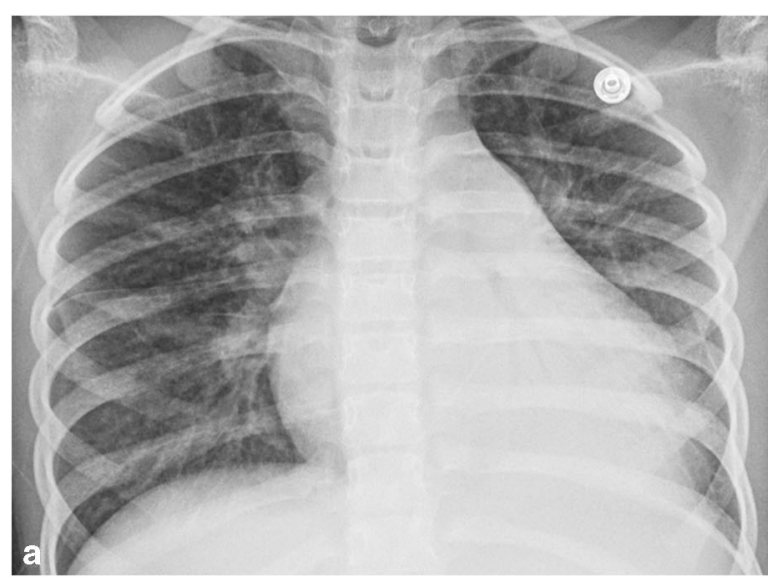

Fig. 2 Chest radiographs in an 8-year-old previously healthy girl who presented with fever, abdominal pain, vomiting, diarrhea and sore throat and rapidly progressed to hypotensive shock and adult respiratory distress syndrome. She was diagnosed with multisystemic inflammatory syndrome in children (MIS-C) with acute myocardial injury. a Anteroposterior (AP) chest radiograph from hospital Day 4

the published data on COVID-19 are on adults. Recent reports from various countries affected by the pandemic reveal that 1.4-5.6\% of COVID-19 cases occur in children. Most of these children are asymptomatic or mildly ill, while $3.5 \%$ and $0.2 \%$ of affected children are severely ill or critically ill, respectively [10]. In a large review of 2,135 children with confirmed or presumed COVID-19 infection, most were mildly ill (51\%) while only $5.9 \%$ were reported as severely ill [11].

Symptomatic children present with fever and a variety of other complaints including headache, diarrhea, sore throat, cough and nasal congestion. As in our study group, many of those requiring admission present with a respiratory illness characterized by respiratory distress, cough and fever $[12,13]$.

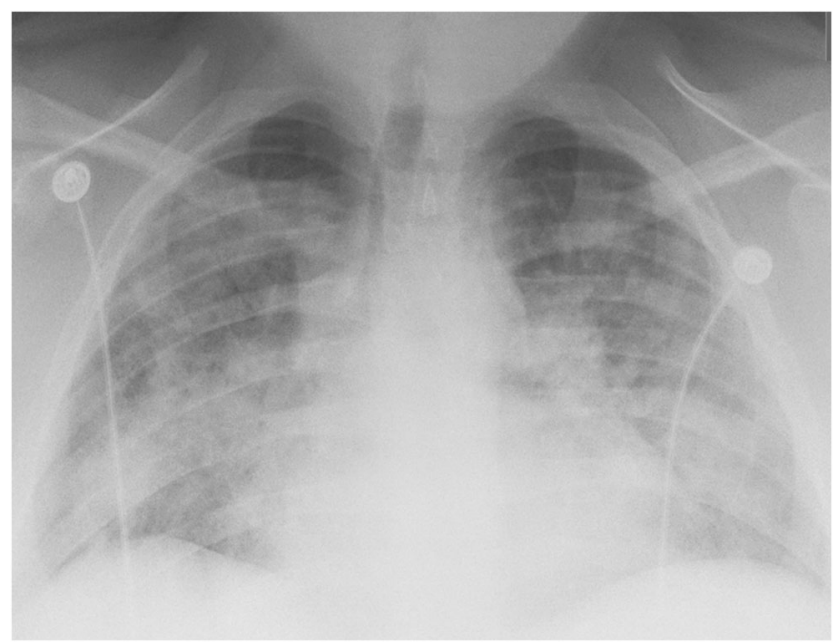

Fig. 3 Admission anteroposterior chest radiograph in a 17-year-old boy with a history of comorbidities. He presented with fever, cough, shortness of breath and headache and was diagnosed with COVID-19 pneumonia. Note the bilateral pulmonary opacities with a predominant perihilar and basilar distribution

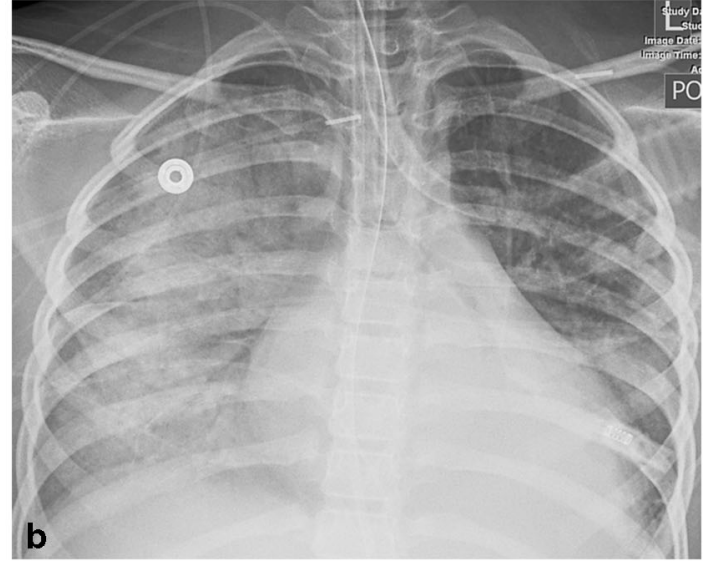

demonstrates cardiomegaly, mild interstitial pulmonary edema and left lower lobe atelectasis. b AP chest radiograph obtained $8 \mathrm{~h}$ later demonstrates interval development of diffuse bilateral air-space opacities consistent with the clinical diagnosis of adult respiratory distress syndrome. The girl required intubation

Unlike the adult population, few children with COVID-19 require ventilatory support. In a review of 41 children younger than 18 years who presented to a Madrid emergency room with COVID-19, $60 \%$ were hospitalized and only $16 \%$ of those required respiratory assistance beyond the use of a nasal cannula for supplementary oxygen [13].

Of the patients in our study group, 63\% required intubation or high-flow nasal cannula therapy on admission; twothirds had parenchymal lung disease. The high rate of respiratory failure in our study group might reflect the increased incidence of pre-existing medical conditions in our patient population. Sixty-three percent of patients in our study had pre-existing conditions, compared to $25 \%$ reported by Tagarro et al. [13] and 27\% reported by Parri et al. [12]. It has been suggested that the incidence of intubation in COVID-19-positive children is higher in those with preexisting medical conditions $[11,13]$. However, this is not uniformly reported [12].

Recently, cardiovascular manifestations of COVID-19 infection in adults have been reported. COVID-19 binds to cells expressing angiotensin-converting enzyme 2 (ACE2) receptors found on cardiomyocytes [6]. Acute cardiac injury, defined as elevation of cardiac troponin above the 99th percentile, is the most common cardiac abnormality seen in adults, occurring in $8-12 \%$ of COVID-19-positive patients. Causes of acute cardiac injury are most often related to viral myocarditis, although myocardial injury secondary to cytokine storm and multiorgan failure, coronary artery thrombosis, and hypoxia can occur [14].

To date, to our knowledge, the incidence of COVID-19associated myocarditis or other myocardial injury in children has not been reported. Cui et al. [15] reported a 55-day-old infant with mild CT findings of pneumonia who demonstrated elevated troponin on admission, indicating myocardial injury. 
Table 2 Type and distribution of pulmonary opacities in pediatric patients with parenchymal lung disease

\begin{tabular}{|c|c|c|c|c|c|c|c|}
\hline \multirow[b]{2}{*}{$\begin{array}{l}\text { Age } \\
\text { (years) }\end{array}$} & \multicolumn{3}{|c|}{ Opacity type } & \multicolumn{4}{|c|}{ Opacity location } \\
\hline & Interstitial & $\begin{array}{l}\text { Hazy } \\
\text { opacities }\end{array}$ & Consolidation & $\begin{array}{l}\text { Lower } \\
\text { lobes }\end{array}$ & Perihilar & Peripheral & Diffuse \\
\hline 11 & $\mathrm{x}$ & $\mathrm{x}$ & $\mathrm{x}$ & & $\mathrm{x}$ & & \\
\hline 0.25 & & $\mathrm{x}$ & $\mathrm{x}$ & $\mathrm{x}$ & $\mathrm{x}$ & & $\mathrm{x}$ \\
\hline 14 & & & $\mathrm{x}$ & $\mathrm{x}$ & $\mathrm{x}$ & & \\
\hline 14 & $\mathrm{x}$ & $\mathrm{x}$ & $\mathrm{x}$ & & & & $\mathrm{x}$ \\
\hline 16 & & & $\mathrm{x}$ & $\mathrm{x}$ & $\mathrm{x}$ & & \\
\hline 18 & & & $\mathrm{x}$ & & & & $\mathrm{x}$ \\
\hline 17 & & & $\mathrm{x}$ & $\mathrm{x}$ & $\mathrm{x}$ & & \\
\hline 4 & & $\mathrm{x}$ & $\mathrm{x}$ & $\mathrm{x}$ & $\mathrm{x}$ & & \\
\hline 15 & $\mathrm{x}$ & & $\mathrm{x}$ & $\mathrm{x}$ & $\mathrm{x}$ & & \\
\hline 14 & $\mathrm{x}$ & & $\mathrm{x}$ & & & $\mathrm{x}$ & \\
\hline 17 & $\mathrm{x}$ & & & & $\mathrm{x}$ & & \\
\hline 7 & $\mathrm{x}$ & $\mathrm{x}$ & & & $\mathrm{x}$ & & $\mathrm{x}$ \\
\hline 8 & & $\mathrm{x}$ & $\mathrm{x}$ & $\mathrm{x}$ & & & $\mathrm{x}$ \\
\hline 3 & & $\mathrm{x}$ & $\mathrm{x}$ & $\mathrm{x}$ & $\mathrm{x}$ & & \\
\hline 4 & & $\mathrm{x}$ & $\mathrm{x}$ & $\mathrm{x}$ & $\mathrm{x}$ & & \\
\hline Total $^{\mathrm{a}}$ & 6 & 8 & 13 & 9 & 11 & 1 & 5 \\
\hline
\end{tabular}

${ }^{a}$ This table includes 15 patients with parenchymal lung disease. The table excludes three patients diagnosed with myocarditis with no parenchymal lung disease and one 3-month-old who presented with fever and respiratory symptoms and had normal chest radiographs
The pathogenesis of COVID-19-related myocardial injury in children is not known. It has been suggested that children are less affected by the virus because viral binding of ACE2 in children is lower than that in adults [11]. Thus, myocarditis in children might not reflect ACE2 viral binding but might rather be related to an associated immune host response. Recent reports describe a new syndrome of multisystem inflammation in children associated with COVID-19 (MIS-C). The syndrome exhibits some features that overlap with Kawasaki disease and others that overlap with toxic shock syndrome. Patients typically present with prolonged fever, gastrointestinal symptoms and mucocutaneous manifestations and develop multisystem inflammation primarily affecting the cardiovascular system $[9,16]$. Of the patients with acute myocardial injury in our cohort, three were subsequently diagnosed with MIS-C. Of these three, one exhibited cardiac dysfunction and ectatic coronary arteries on an echocardiogram, and developed adult respiratory distress syndrome; the other two demonstrated cardiac dysfunction with no dilatation of coronary arteries and did not have parenchymal lung disease on chest radiographs. The development of acute cardiac injury is a poor prognostic indicator in adults [14]. The outcome of COVID19-related myocardial disease in children has not been determined.

The radiographic findings in young children $<10$ years with COVID-19 and myocarditis differed from those of older children who did not develop myocarditis. Children with myocarditis demonstrated cardiomegaly, pulmonary vascular congestion or interstitial edema, and pleural effusion on chest radiographs. There was a notable absence of air-space opacities. In contrast, $93 \%$ of children without myocardial injury demonstrated pulmonary consolidations or hazy parenchymal infiltrates on chest radiographs.

Unlike adults with COVID-19, who often present with peripheral and basilar parenchymal opacities [17], the pulmonary findings in our study group were more often perihilar in location. In only one child was the distribution of parenchymal abnormalities peripheral. As in adults, lower lobe predominance was common and upper lobe disease was absent except in those who had diffuse opacities. Pleural effusions were also uncommon in this group of patients.

Our study is limited by the small number of patients, reflecting the lower incidence and milder presentation of COVID-19 in children. Because of the small sample size, pediatric radiologists were not blinded to the clinical diagnosis and thus observer bias might be present. Additionally, because respiratory virus panel testing was performed in only half of patients in the cohort, it is possible that other viral infections contributed to the radiographic findings. Last, only patients who were admitted to the hospital were included in the study.

\section{Conclusion}

In summary, we present our findings in children with COVID19 who were admitted to a hospital. In our small cohort, 12 
children presented with respiratory failure and required intubation or high-frequency nasal cannula therapy. The majority had comorbidities. Two children, both with comorbidities, died during the study period.

Children in their first decade developed myocarditis as a component of a newly described syndrome of multisystemic inflammation (MIS-C). These children exhibited cardiomegaly, pulmonary vascular congestion or interstitial pulmonary edema, and small pleural effusions on chest radiographs, as opposed to children in their second decade in whom pulmonary disease dominated the clinical and radiographic features of COVID19. In these children pulmonary opacities demonstrated predominantly a perihilar and basilar distribution unlike in adults, who tend to exhibit more peripheral opacities.

MIS-C is a newly described syndrome of multisystem inflammation in children and its association with COVID-19 requires further investigation.

\section{Compliance with ethical standards}

Conflicts of interest None

\section{References}

1. Park SE (2020) Epidemiology, virology, and clinical features of severe acute respiratory syndrome-coronavirus-2 (SARS-CoV-2; coronavirus disease-19). Clin Exp Pediatr. https://doi.org/10.3345/ cep. 2020.00493

2. Worldometer (2020) Coronavirus cases: 10,500,639; deaths: 510, 736. https://www.worldometers.info/coronavirus/. Accessed 30 June 2020

3. Cao Y, Liu X, Xiong L, Cai K (2020) Imaging and clinical features of patients with 2019 novel coronavirus SARS-CoV-2: a systematic review and meta-analysis. J Med Virol. https://doi.org/10.1002/jmv. 25822

4. Durvasula R, Wellington T, McNamara E, Watnick S (2020) COVID-19 and kidney failure in the acute care setting: our experience from Seattle. Am J Kidney Dis 76:4-6
5. Bikdeli B, Madhavan V, Jimenez D et al (2020) COVID-19 and thrombotic or thromboembolic disease: implications for prevention, antithrombotic therapy, and follow-up. JACC state-of-the-art review. J Am Coll Cardiol 75:2950-2973

6. Xiong TY, Redwood S, Prendergast B, Chen M (2020) Coronaviruses and the cardiovascular system: acute and longterm implications. Eur Heart J 41:1798-1800

7. She J, Liu L, Liu W (2020) COVID-19 epidemic: disease characteristics in children. J Med Virol 92:747-754

8. Mallineni SK, Innes NP, Raggio DP et al (2020) Coronavirus disease (COVID-19): characteristics in children and considerations for dentists providing their care. Int J Paediatr Dent 30:245-250

9. Riphagen S, Gomez X, Gonzalez-Martinez C et al (2020) Hyperinflammatory shock in children during COVID-19 pandemic. Lancet 395:1607-1608

10. Choi SH, Kim HW, Kang JM et al (2020) Epidemiology and clinical features of coronavirus disease 2019 in children. Clin Exp Pediatr 63:125-132

11. Dong Y, Mo X, Hu Y et al (2020) Epidemiology of COVID-19 among children in China. Pediatrics 145:e20200702

12. Parri N, Lenge M, Buonsenso D (2020) Children with Covid-19 in pediatric emergency departments in Italy. N Engl J Med. https://doi. org/10.1056/NEJMc2007617

13. Tagarro A, Epalza C, Santos M et al (2020) Screening and severity of coronavirus disease 2019 (COVID-19) in children in Madrid. Spain JAMA Pediatr. https://doi.org/10.1001/jamapediatrics.2020. 1346

14. Bansal M (2020) Cardiovascular disease and COVID-19. Diabetes Metab Syndr 14:247-250

15. Cui Y, Tian M, Huang D et al (2020) A 55-day-old female infant infected with 2019 novel coronavirus disease: presenting with pneumonia, liver injury, and heart damage. J Infect Dis 221: $1775-1781$

16. Jones VG, Mills M, Suarez D et al (2020) COVID-19 and Kawasaki disease: novel virus and novel case. Hosp Pediatr 10: $537-540$

17. Wong HYF, Lam HYS, Fong AH et al (2019) Frequency and distribution of chest radiographic findings in COVID-19 positive patients. Radiology 2020:201160

Publisher's note Springer Nature remains neutral with regard to jurisdictional claims in published maps and institutional affiliations. 